\title{
Efecto de dos abonos orgánicos en las cadenas tróficas del suelo cultivado con mora ${ }^{1}$
}

\section{Effect of two organic fertilizers on food webs of soil cultivated with blackberry}

\author{
Martha Orozco-Aceves ${ }^{2}$, José Alonso Calvo-Araya ${ }^{3}$, Jean Alexander Gamboa-Tabares ${ }^{4}$, Wálter Peraza-Padilla ${ }^{5}$, \\ Orlado Varela-Rodríguez ${ }^{6}$, Rafael Orozco-Rodríguez ${ }^{7}$
}

\begin{abstract}
Resumen
La adición de abonos orgánicos es una práctica que mejora las propiedades biológicas del suelo; sin embargo, los efectos de estos sobre las cadenas tróficas (CT) del suelo han sido poco estudiados. El objetivo del trabajo fue determinar el efecto de dos abonos orgánicos comerciales sobre la estructura de las CT asociadas a raíces de plantas de mora (Rubus adenotrichos). La investigación se realizó en dos plantaciones de mora en San Martín de León Cortés y Buena Vista de Pérez Zeledón, San José, Costa Rica, durante agosto-diciembre del 2010. En ambas plantaciones las plantas fueron fertilizadas con compost o lombricompost. Se muestreó el suelo que circundaba las raíces de las plantas para cuantificar los grupos de organismos de las CT, mediante las siguientes técnicas: bacterias y hongos filamentosos mediante cuenta directa en placa, protozoarios mediante el número más probable, esporas de hongos micorrizógenos y nematodos mediante flotación-centrifugación, microartrópodos, macroartrópodos y lombrices fueron cuantificados directamente en el suelo. Los resultados fueron analizados mediante análisis de escalamiento multidimensional. La adición de abonos orgánicos causó un efecto diferencial sobre la estructura de las CT (con respecto a suelos sin fertilizar). Dependiendo del tipo de abono, este efecto varió en cada una de las fincas. Los grupos de organismos mayormente afectados fueron actinomicetes y protozoarios, lo cual implica que la estructura de las CT y como consecuencia, las funciones del suelo no fueron afectadas por la adición de abonos orgánicos.
\end{abstract}

Palabras clave: agricultura orgánica, biología del suelo, compost, lombricompost, Rubus adenotrichos.

\begin{abstract}
Soil fertilization with organic fertilizers comprises a practice that improves the soil biological properties; however, the effect of these on the soil food web (SFW) has been scarcely studied. The aim of this study was to determine the
\end{abstract}

1 Recibido: 9 de agosto, 2016. Aceptado: 1 de febrero, 2017. Este trabajo fue parte de un proyecto de investigación en el marco de la Maestría en Agricultura Alternativa con mención en Agricultura Ecológica, impartida en la Universidad Nacional. Heredia, Costa Rica.

2 Universidad Nacional, Instituto Regional de Estudios en Sustancias Tóxicas. Apartado postal 86-3000 Heredia, Costa Rica. marthaorozcoaceves@gmail.com

3 Universidad Nacional, Escuela de Ciencias Agrarias, Laboratorio de Fitopatología. Apartado postal 86-3000 Heredia, Costa Rica. josealonsocalvoaraya@gmail.com

4 Universidad de la Amazonía, Laboratorio de Entomología. Florencia, Caquetá, Colombia. gamboatabares@gmail.com

5 Universidad Nacional, Escuela de Ciencias Agrarias, Laboratorio de Nematología. Apartado postal 86-3000 Heredia, Costa Rica. walter. peraza.padilla@una.cr

6 Universidad Nacional, Escuela de Ciencias Agrarias, Laboratorio de Recursos Fitogenéticos. Apartado postal 86-3000 Heredia, Costa Rica. ovarelar@una.cr

7 Universidad Nacional, Escuela de Ciencias Agrarias, Laboratorio de Genética Vegetal y Propagación de Plantas. Apartado postal 86-3000 Heredia, Costa Rica. Rafael.orozco.rodriguez@una.cr 
effect of two commercial organic fertilizers on the structure of the SFW associated with roots of blackberry plants (Rubus adenotrichos). The research was conducted in two blackberry plantations located one in San Martín de León Cortés, and the other one in Buena Vista de Pérez Zeledón in San José, Costa Rica, from August to December, 2010. In the two plantations, plants were fertilized with compost or vermicompost. The roots of blackberry plants surrounding soil were sampled in order to quantify groups of the SFW through the following techniques: bacteria and filamentous fungi by plate count, protozoa by the most probable number, spores from arbuscular mycorrhizal fungi and nematodes by flotation-centrifugation, microarthropods, macroarthropods, and worms were directly counted in soil samples. The dataset was analyzed by multidimensional scaling analysis. The addition of organic fertilizers to soil caused a differential effect on the structure of the SFW (as compared with non-fertilized soils). The effect differed in soil from each of the experimental plantations according to fertilizer type. The groups of organisms mainly affected were actinomycetes and protozoa, which implies that the structure of SFW and consequently, the function of soil were not affected by the addition of organic fertilizers.

Keywords: organic agriculture, soil biology, compost, vermicompost, Rubus adenotrichos.

\section{Introducción}

El uso de abonos orgánicos en la producción agrícola es considerado una práctica sustentable, ya que conserva y mejora las propiedades físicas y químicas del suelo (Meléndez, 2003; Soto, 2003; van-der-Heijden y Wagg, 2013). Además, ha sido demostrado que los abonos orgánicos favorecen el desarrollo de la biota del suelo, lo cual es deseable en un agroecosistema, debido a los efectos positivos de los organismos edáficos en la producción vegetal (Altieri, 1999; Wardle, 1999; Liu et al., 2009; Kallenbach y Grandy, 2011; Steel et al., 2012; Wu et al., 2013). Sin embargo, en el suelo, los grupos de organismos no se organizan aisladamente, sino que forman cadenas tróficas (CT), las cuales son redes de interacciones presa-consumidor que se presentan entre grupos de organismos, poblaciones, o unidades tróficas agregadas (Smeding y de-Snoo, 2003; de-Vries et al., 2013). En el suelo, las CT son responsables de la disponibilización de nutrientes para las plantas, influyen directamente en la movilización de materia y energía en el suelo y, por consiguiente, son críticas para el funcionamiento de los ecosistemas (Wardle, 1999). En plantaciones de mora, diversos tipos de microorganismos de las CT, como los hongos micorrizógenos, bacterias solubilizadoras de fosfatos y bacterias fijadoras de nitrógeno, influyen directamente en la productividad de las plantas. Estos microorganismos incluso han sido propuestos para utilizarse como biofertilizantes en dicho cultivo (Roveda et al., 2008).

Las prácticas agrícolas disturban la organización espacial y temporal del suelo, afectando negativamente la estructura de las CT y modifican la dinámica de nutrientes (Moore, 1994; Noble y Ruaysoongnern, 2010; Tilston et al., 2010; de-Vries et al., 2013). La adición de abonos orgánicos al suelo es aceptada como una práctica amigable con el ambiente; sin embargo, existe la posibilidad de que su adición produzca alteraciones en las CT. Por ejemplo, ha sido demostrado que la adición de mulch de rastrojo de paja al suelo activa el canal de descomposición bacteriana en detrimento del canal de descomposición fúngico (Moore y Hunt, 1988; Moore, 1994). Este hallazgo indica que la adición de abonos orgánicos puede afectar la estructura y la función de las CT, con implicaciones en la tasa de descomposición de la materia orgánica y del funcionamiento del suelo en general.

El uso de abonos orgánicos como compost y lombricompost constituye una alternativa viable al uso de fertilizantes químicos, debido al encarecimiento de estos últimos, además del interés por parte de productores y consumidores de promover una producción sustentable y limpia, conservar el ambiente y la salud humana (Gliessman, 2002). En Costa Rica, los abonos orgánicos están siendo utilizados principalmente por pequeños productores, quienes han dirigido su interés hacia la producción de cultivos no tradicionales como la mora dulce 
(Rubus adenotrichos cv. 'Vino'; Orozco y Muñoz, 2012). Sin embargo, para confirmar que la incorporación de abonos orgánicos al suelo es una práctica que conserva y mejora las propiedades biológicas del suelo, es deseable investigar los efectos de estos abonos sobre las CT. El objetivo del trabajo fue determinar el efecto de dos abonos orgánicos comerciales sobre la estructura de las CT asociadas a raíces de plantas de mora.

\section{Materiales y métodos}

\section{Diseño experimental y sitios muestreados}

\section{Zona de estudio y condiciones ambientales}

El estudio se realizó durante agosto-diciembre del 2010 en dos plantaciones de mora (Rubus adenotrichos) con seis años de establecimiento, una localizada en San Martín de León Cortés (SMLC; 09³0`15,5” N, 083³9`28,7" $\mathrm{W}$, altitud de $1713 \mathrm{~m}$, área de $3363 \mathrm{~m}^{2}$, precipitación promedio anual de $3379 \mathrm{~mm}$, temperaturas máxima y mínima de 26,4 y $14,3{ }^{\circ} \mathrm{C}$, humedad relativa máxima y mínima de 86,01 y $56,4 \%$, respectivamente) y otra, en Buena Vista de Pérez Zeledón (BVPZ; 0943`44,0” N, 08400`05,1” W, altitud de $1860 \mathrm{~m}$, área de 2312 m², precipitación promedio de $2618 \mathrm{~mm}$, temperaturas máxima y mínima de 27,6 y $12,1^{\circ} \mathrm{C}$, humedad máxima y mínima de 93,63 y 41,7\%) (Orozco y Rosales, 2009), ambas localidades pertenecientes a la provincia de San José, Costa Rica.

En las plantaciones de mora la densidad de siembra fue de 333 plantas/ha. El diseño experimental consistió en bloques completos al azar. En BVPZ se establecieron cuatro bloques y en SMLC se establecieron tres. En cada bloque, las unidades experimentales estuvieron constituidas por cuatro plantas en competencia completa, sembradas en una hilera. Entre unidades experimentales sucesivas del mismo bloque se dejó una planta de borde, no así entre bloques. En cada bloque, las unidades experimentales recibieron uno de dos tratamientos de fertilización orgánica: abonado con compost (4 kg por planta) o con lombricompost (3 kg por planta); además, se establecieron controles conformados por plantas sin fertilizar. Para el muestreo, se seleccionaron al azar tres plantas por tratamiento por bloque, de las cuales se tomó el suelo que circundaba la raíz a $20 \mathrm{~cm}$ de profundidad; por cada planta se tomó aproximadamente $1 \mathrm{~kg}$ de suelo. Las muestras de suelo fueron almacenadas a $4{ }^{\circ} \mathrm{C}$ hasta su procesamiento en los laboratorios de Ecología de suelos y Nematología de la Escuela de Ciencias Agrarias de la Universidad Nacional.

\section{Determinación de las características físico-químicas de los suelos}

En SMLC el suelo fue clasificado como: orden Inceptisol, suborden Andepts, gran grupo Dystrandepts; mientras que en BVPZ se clasificó como: orden Ultisol, suborden Humults, gran grupo Tropohumults (Soil Taxonomy, 1999; Ortiz, 2004). En SMLC el suelo presentó un pH de 4,4; acidez intercambiable de 4,4 cmol (+)/1; cantidad de materia orgánica de 9,6\%; concentraciones de $\mathrm{P}$ de $3,3 \mathrm{mg} / 1 ; 0,34,0,2$ y 0,19 cmol (+)/l de Ca, $\mathrm{Mg}$ y $\mathrm{K}$, respectivamente; 1,0, 2,0, 230 y 5,0 mg/l de Zn, Cu, Fe y Mn; textura franca (43,8\% de arena, 34,6\% de limo y 21,6\% de arcilla). En BVPZ el suelo presentó las siguientes características físico-químicas: pH de 5,3; acidez intercambiable de 1,1 cmol (+)/l; cantidad de materia orgánica de10,3\%; concentración de P de 3,0 mg/l; 3,75, 0,7 y $0,25 \mathrm{cmol}(+) / \mathrm{l}$ de $\mathrm{Ca}, \mathrm{Mg}$ y K, respectivamente; 7,0, 5,0, 165,0 y 33,0 mg/l de Zn, Cu, Fe y Mn, respectivamente; textura franca (49,8\% de arena, 32,6\% de limo y 17,6\% de arcilla; Orozco y Muñoz, 2012). 


\section{Abonos orgánicos}

Los abonos orgánicos utilizados durante el experimento fueron de origen comercial. La composición de ambos se describen en el Cuadro 1.

Cuadro 1. Composición química de abonos orgánicos utilizados en dos plantaciones de mora, localizadas en San Martín de León Cortés y Buena Vista de Pérez Zeledón, San José, Costa Rica. Octubre, 2010.

Table 1. Chemical composition of organic fertilizers applied to two different blackberry plantations located in San Martín de León Cortés and Buena Vista de Pérez Zeledón, San José, Costa Rica. October 2010.

\begin{tabular}{ccc}
\hline Propiedad & Compost & Lombricompost \\
\hline Humedad (\%) & 44 & 36 \\
$\mathrm{pH}\left(\mathrm{H}_{2} \mathrm{O} 1: 2,5\right)$ & 7,3 & 8,5 \\
Materia orgánica $(\%)$ & 13,8 & 34,4 \\
$\mathrm{~N}(\%)$ & 1,14 & 2,55 \\
$\mathrm{C}(\%)$ & 8 & 20 \\
$\mathrm{C}: \mathrm{N}$ & 7,0 & 7,8 \\
$\mathrm{P}(\%)$ & 0,35 & 0,27 \\
$\mathrm{~K}(\%)$ & 0,61 & 2,07 \\
$\mathrm{Ca}(\%)$ & 0,72 & 1,68 \\
$\mathrm{Mg}(\%)$ & 0,17 & 0,38 \\
$\mathrm{Fe}(\mathrm{mg} / \mathrm{kg})$ & 49259 & 29869 \\
$\mathrm{Cu}(\mathrm{mg} / \mathrm{kg})$ & 116 & 85 \\
$\mathrm{Zn}(\mathrm{mg} / \mathrm{kg})$ & 105 & 64 \\
$\mathrm{Mn}(\mathrm{mg} / \mathrm{kg})$ & 627 & 496 \\
\hline
\end{tabular}

Fuente/Source: Orozco y Muñoz (2012).

\section{Variables de respuesta}

En las muestras de suelo que circundaban las raíces de las plantas de mora, se cuantificaron los siguientes organismos pertenecientes a las CT: bacterias aerobias incluyendo actinomicetes, hongos filamentosos, esporas de hongos micorrizógenos, protozoarios, nematodos, microartrópodos, macroartrópodos y lombrices. Las bacterias aerobias y hongos filamentosos se determinaron mediante cuenta directa en placa, para lo cual se realizaron diluciones decimales de suelo (Alef, 1995). Las bacterias aerobias y actinomicetes se cuantificaron en los medios de cultivo agar glucosa-peptona, agar extracto de malta-rosa de bengala y agar caseína-peptona-almidón con extracto de levadura. Los hongos filamentosos se cuantificaron en los medios agar papa-dextrosa y agar Sabouraud-dextrosa. Los resultados de la cuantificación de bacterias aerobias y hongos filamentosos se reportaron en UFC/g suelo.

Para la cuantificación de esporas de hongos micorrizógenos se utilizó la metodología de flotación-centrifugación en solución de azúcar (Bagyaraj y Stürmer, 2008), los resultados se reportaron en número de esporas/g suelo. Los 
protozoarios se determinaron mediante la técnica del número más probable (NMP) (Alef, 1995; Coleman et al., 2004), y para realizar los cálculos correspondientes se utilizó el programa 'MPN Calculator'. Los resultados se reportaron en NMP de protozoarios/g suelo.

Los nematodos se extrajeron mediante la metodología de centrifugación-flotación en solución de azúcar (Cares y Huang, 2008). Los nematodos totales fueron cuantificados, así como el número de nematodos de los diferentes grupos tróficos (bacteriófagos, fitófagos, micófagos, depredadores y omnívoros), los cuales fueron asignados con base en los criterios de Yeates et al. (1993). Los resultados se reportaron en nematodos/g suelo y los diferentes grupos tróficos se expresaron en porcentajes.

Los microartrópodos fueron extraídos de $100 \mathrm{~g}$ de suelo mediante embudos de Berlesse (Karyanto et al., 2008), para posteriormente cuantificarlos. Los macroartrópodos fueron extraídos manualmente de $1 \mathrm{~kg}$ de suelo y cuantificados (Huising et al., 2008). Finalmente, para determinar el número de lombrices se trazaron en el terreno cuadrantes de $25 \times 25 \mathrm{~cm}$, el suelo dentro del cuadrante fue tomado a una profundidad de $30 \mathrm{~cm}$. El número de lombrices se contó y se reportó en individuos/m² (Bignell et al., 2008; Huising et al., 2008).

\section{Análisis de resultados}

Los resultados de la cuantificación de los organismos de las CT circundando la raíz de las tres plantas muestreadas por tratamiento de fertilización orgánica fueron promediados. Con los datos se realizó un análisis de escalamiento multidimensional (MDS) (Clarke y Gorley, 2006), para determinar diferencias en la estructura de las CT de acuerdo con los tratamientos de fertilización en ambas fincas. Para construir el MDS, los datos fueron normalizados y se utilizó la distancia Euclidiana. En el MDS se superpuso un vector indicando los grupos de organismos cuyas diferencias causaron la mayor separación de los tratamientos en el espacio multidimensional; dicho vector muestra los grupos de organismos cuyo coeficiente de correlación de Pearson con MDS1 y MDS2 fue $>0,7$. Finalmente, se realizó un análisis de conglomerados para determinar los tratamientos en donde la estructura de las CT del suelo eran similares entre sí.

\section{Resultados y discusión}

La adición de los abonos orgánicos, compost y lombricompost tuvo un efecto diferencial sobre las CT circundando raíces de plantas de mora. Dicho efecto varió en cada una de las fincas de acuerdo con las características particulares del suelo en las mismas. El análisis multidimensional (MDS) mostró que los suelos de las dos fincas experimentales eran biológicamente diferentes, por lo tanto, los suelos se separaron a lo largo del MDS1 de acuerdo con el tipo de finca (Figura 1). Los suelos de SMLC se distribuyeron en el lado izquierdo del gráfico, mientras que los suelos de BVPZ se distribuyeron en el lado derecho (Figura 1). Este resultado es lógico, debido a la heterogeneidad inherente a los suelos de cada localidad. Los grupos de organismos que difirieron de acuerdo con el tipo de finca, es decir, que tuvieron un coeficiente de correlación de Pearson con el MDS1 > 0,7, fueron las bacterias aerobias totales $(-0,87)$, macroartrópodos $(-0,76)$, nematodos fitófagos $(-0,98)$, nematodos totales $(-0,99)$, lombrices $(-0,76)$, nematodos micófagos $(0,93)$, nematodos bacteriófagos $(0,98)$ y nematodos omnívoros $(0,95 ;$ Figura 1$)$. El número de bacterias aerobias, nematodos totales, nematodos fitófagos, macroartrópodos y lombrices fue mayor en suelos de la finca SMLC; mientras que el número de nematodos bacteriófagos, micófagos y omnívoros fue mayor en la finca BVPZ (Cuadro 2). 


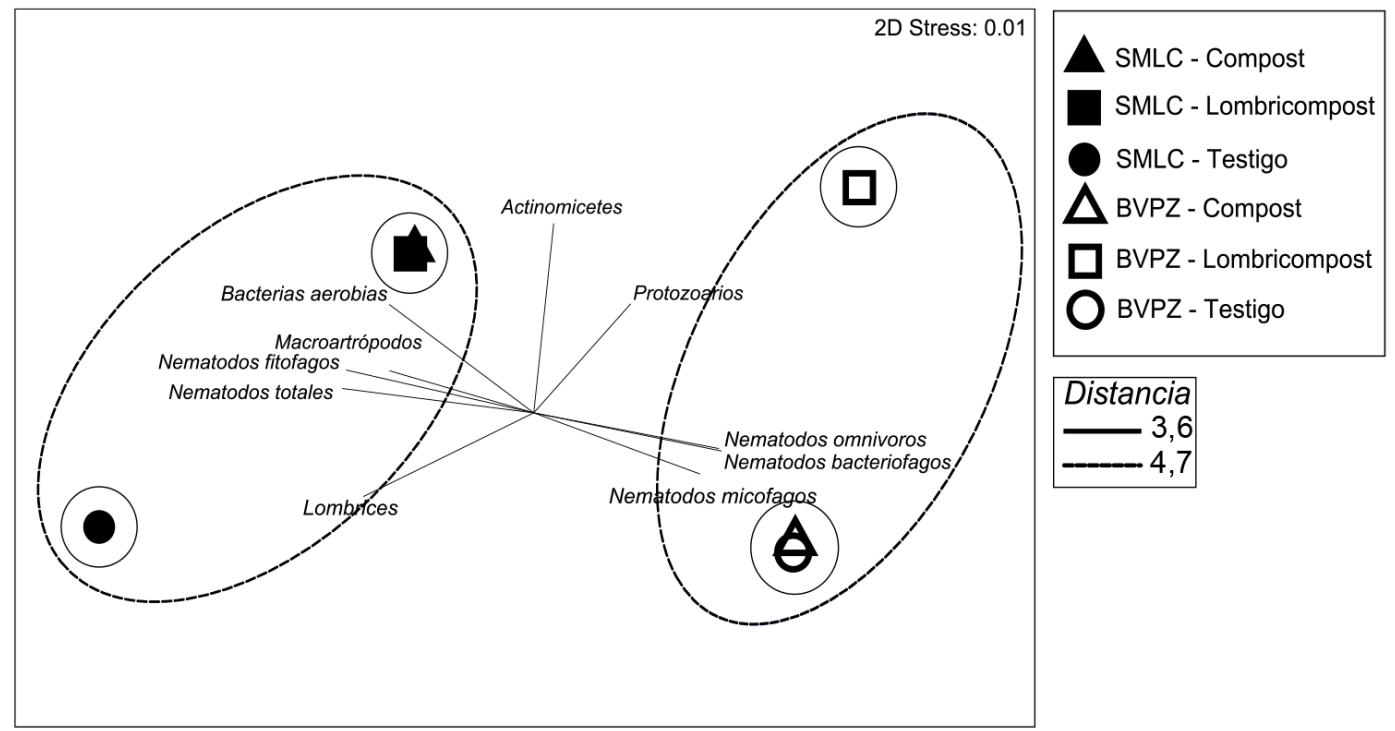

Figura 1. Análisis de escalamiento multidimensional de los organismos de las cadenas tróficas asociadas a raíces de plantas de mora, fertilizadas con abonos orgánicos: compost y lombricompost, y sin fertilizar (controles), en dos localidades, San Martín de León Cortés (SMLC) y Buena Vista de Pérez Zeledón (BVPZ), San José, Costa Rica. Octubre, 2010.

El vector superpuesto sobre el gráfico indica los grupos de organismos cuyo coeficiente de correlación de Pearson con MDS1 y MDS2 es > 0,7. El recuadro inferior derecho indica las distancias calculadas durante el análisis de conglomerados.

Figure 1. Multidimensional scaling analysis of the organisms of the soil food web associated with roots of blackberry plants that were fertilized with organic fertilizers: compost and vermicompost, and with no fertilization (controls), in two locations, San Martín de León Cortés (SMLC) and Buena Vista de Pérez Zeledón (BVPZ), San José, Costa Rica. October, 2010. The overlaid vector on the plot shows the groups of organisms with Pearson correlation coefficient with MDS1 and MDS2 $>0,7$. The lower right box indicates the calculated distance during the cluster analysis.

Cuadro 2. Resultados de la cuantificación de organismos de las cadenas tróficas asociadas a raíces de plantas de mora bajo dos tratamientos de fertilización orgánica y plantas control sin fertilizar. San Martín de León Cortés y Buena Vista de Pérez Zeledón, San José, Costa Rica. Octubre, 2010.

Table 2. Results of organisms quantification of the soil food webs associated with roots of blackberry plants that were fertilized with organic fertilizers, and results from non-fertilized control plants. San Martín de León Cortés and Buena Vista de Pérez Zeledón, San José, Costa Rica. October 2010.

\begin{tabular}{lcccccc}
\hline \multirow{2}{*}{ Grupo } & \multicolumn{3}{c}{ Finca San Martín de León Cortés } & \multicolumn{3}{c}{ Finca Buena Vista de Pérez Zeledón } \\
\cline { 2 - 7 } & Control & Compost & Lombricompost & Control & Compost & Lombricompost \\
\hline Bacterias aerobias totales (UFC/g suelo) & $2,3 \times 10^{10}$ & $1,15 \times 10^{10}$ & $5 \times 10^{9}$ & $3,5 \times 10^{9}$ & $5,2 \times 10^{9}$ & $2,5 \times 10^{8}$ \\
Actinomicetes (UFC/g suelo) & $3 \times 10^{8}$ & $1 \times 10^{9}$ & $2 \times 10^{8}$ & $9,5 \times 10^{8}$ & $7 \times 10^{8}$ & $2,5 \times 10^{8}$ \\
Hongos filamentosos (UFC/g suelo) & $1 \times 10^{6}$ & $2 \times 10^{8}$ & $1 \times 10^{7}$ & $4,5 \times 10^{6}$ & $5 \times 10^{6}$ & $7 \times 10^{6}$ \\
Hongos micorrizógenos (esporas/g suelo) & 99,19 & 27,49 & 39,38 & 25,58 & 15,34 & 102,31 \\
Protozoarios (NMP/g suelo) & $16,2 \times 10^{3}$ & $11,4 \times 10^{3}$ & $4,2 \times 10^{3}$ & $16,8 \times 10^{3}$ & $28 \times 10^{3}$ & $11 \times 10^{3}$ \\
Nematodos totales (individuos/g suelo) & 2,65 & 2,35 & 2,33 & 1,64 & 1,56 & 1,54 \\
Nematodos bacteriófagos (\%) & 4 & 17 & 10 & 31 & 36 & 38 \\
Nematodos micófagos (\%) & 0 & 3 & 2 & 5 & 4 & 4 \\
Nematodos fitófagos (\%) & 96 & 79 & 87 & 60 & 56 & 50 \\
Nematodos depredadores (\%) & 0 & 0 & 0 & 0 & 0 & 2 \\
Nematodos omnívoros (\%) & 0 & 1 & 1 & 4 & 4 & 6 \\
Microartrópodos totales (individuos/kg suelo) & 15 & 15 & 6 & 29 & 15 & 8 \\
Macroartrópodos totales (individuos/kg suelo) & 14 & 3 & 16 & 3 & 2 & 3 \\
Lombrices (individuos 0,019 m m $^{3}$ suelo) & 61 & 120 & 85 & 2 & 2 & 12 \\
\hline
\end{tabular}


El efecto de los abonos orgánicos sobre las CT se demostró por el MDS, los tratamientos de fertilización orgánica se separaron a lo largo de MDS2 (Figura 1), pero dicho efecto difirió en los dos suelos experimentales. En suelo de SMLC la adición de los dos abonos produjo cambios en la estructura de las CT, dichos efectos fueron similares entre sí (los tratamientos se ubicaron muy cercanamente en el MSD), pero diferentes de los controles (Figura 1). En BVPZ, únicamente el lombricompost tuvo efecto en las CT, ya que estas difirieron de las CT del suelo fertilizado con compost y de los controles, siendo estas dos últimas similares entre sí. Como consecuencia, los suelos fertilizados con lombricompost se separaron de los suelos fertilizados con compost y de los controles en el MDS (Figura 1).

El MDS no evidenció un efecto de los abonos orgánicos sobre grupos microbianos del suelo como bacterias aerobias totales y hongos filamentosos (con respecto a suelos control sin fertilizar), los cuales son altamente responsivos (aumento en la densidad poblacional) a la adición de materia orgánica (Scheu et al., 2005; Condron et al., 2010; Kramer et al., 2013). Por otro lado, los grupos de organismos de las CT cuyas poblaciones sí fueron afectados por la adición de los abonos orgánicos, es decir, que tuvieron un coeficiente de correlación de Pearson con MDS2 > 0,7, fueron los actinomicetes $(-0,94)$ y los protozoarios $(-0,75)$.

Los actinomicetes se encontraron en mayor número en el suelo fertilizado con compost en SMLC, mientras que en BVPZ, estos se encontraron en mayor número en los controles (Cuadro 2). Las poblaciones de actinomicetes se incrementaron con la adición de abonos orgánicos (Giri et al., 2005; Reilly et al., 2013), por lo que, los resultados obtenidos en el presente estudio contradicen lo reportado en la literatura. Las propiedades de los abonos orgánicos debieron haber influido en los resultados obtenidos. Por ejemplo, el pH es crítico para la reproducción y desarrollo de los microorganismos. Los actinomicetes se desarrollan de manera óptima en un pH de 6,5 a 8,9 (Lavelle y Spain, 2003; Giri et al., 2005). El pH del compost fue de 7,3, por lo que, la adición de este abono pudo haber influido de manera positiva en la población de actinomicetes del suelo de SMLC, al proveer condiciones más favorables para la reproducción de este grupo de organismos. En contraste, el pH del lombricompost fue de 8,5, lo cual pudo haber producido un efecto adverso para la reproducción de los actinomicetes, los cuales presentaron la densidad poblacional más baja en el suelo fertilizado con este abono orgánico en ambas fincas.

Los protozoarios se encontraron en mayor número en los suelos control en SMLC; por otro lado, en BVPZ se presentaron en un número mayor en el suelo fertilizado con compost (Cuadro 2). Las poblaciones de protozoarios fueron negativamente afectadas (con respecto a los suelos control) en suelos fertilizados tanto con compost como con lombricompost en SMLC; mientras que en BVPZ, la población de estos organismos fue mayor en el suelo que se utilizó compost y menor en el que se aplicó lombricompost (con respecto a controles). Los protozoarios se alimentan de hongos y bacterias, de esta manera, regulan las poblaciones de microorganismos en el suelo (Singh y Rengel, 2007). Existe una relación proporcional entre la densidad de microorganismos y la población de protozoarios en el suelo (Scheu et al., 2005). Los resultados de este estudio apoyan la información anterior, ya que las poblaciones de protozoarios presentaron una relación proporcional a la densidad de bacterias totales en todos los casos. Es posible que los abonos hayan tenido un efecto directo sobre la densidad de bacterias totales y por consiguiente, un efecto indirecto sobre las poblaciones de protozoarios. Sin embargo, el análisis estadístico utilizado en el presente estudio no detectó diferencias en las poblaciones de bacterias totales (excepto por la densidad de las poblaciones de actinomicetes) en función del tipo de abono orgánico aplicado.

\section{Conclusiones}

La adición de abonos orgánicos a suelo cultivado con plantas de mora tuvo un efecto diferente en cada una de las fincas. Las propiedades particulares del suelo presente en cada sitio y las propiedades físico-químicas de cada uno de los abonos orgánicos debieron haber influido en el tipo y magnitud del efecto sobre la estructura de las CT. 
El efecto de los abonos orgánicos sobre la estructura de las CT fue de menor magnitud al esperado, ya que únicamente dos (actinomicetes y protozoarios) de los catorce grupos (y subgrupos) de organismos cuantificados fueron afectados en el mediano plazo. Esto implica que la estructura de las CT y por lo tanto, las funciones del suelo no fueron afectadas drásticamente por la adición de abonos orgánicos.

\section{Literatura citada}

Alef, K. 1995. Enrichment, isolation and counting of soil microorganisms. In: K. Alef, and P. Nannipieri, editors, Methods in applied soil microbiology and biochemistry. Academic Press, London, GBR. p. 123-191.

Altieri, M.A. 1999. The ecological role of biodiversity in agroecosystems. Agric. Ecosyst. Environ. 74:19-31.

Bagyaraj, J.D., and S.L. Stürmer. 2008. Arbuscular mycorrhizal fungi. In: M.S. Moreira et al., editors, A handbook of tropical soil biology. Earthscan, London, GBR. p. 131-144.

Bignell, D.E., R. Constantino, A.K. Csuzdi, S.Konaté, J. Louzada, F.X. Susilo, J.E. Tondoh, and R. Zanetti. 2008. Macrofauna. In: M.S. Moreira et al., editors, A handbook of tropical soil biology. Earthscan, London, GBR. p. 43-76.

Cares, J.E., and S.P. Huang. 2008. Soil nematodes. In: M.S. Moreira et al., editors, A handbook of tropical soil biology. Earthscan, London, GBR. p. 97-105.

Clarke, K.R., and R.N. Gorley. 2006. PRIMER v6 User manual/tutorial. PRIMER-E Ltd, GBR.

Coleman, D.A., D.A. Crossley, and P.F. Hendrix. 2004. Fundamentals of soil ecology. $2^{\text {nd }}$ ed. Elsevier Academic Press, Amsterdam, HOL.

Condron, L., C. Stark, M. O'Callaghan, P. Clinton, and Z. Huang. 2010. The role of microbial communities in the formation and decomposition of soil organic matter. In: G.R. Dixon, and E.L. Tilston, editors, Soil microbiology and sustainable crop production. Springer, USA. p. 81-118.

De-Vries, F.T., E. Thébault, M. Liiri, K. Birkhofer, M.A. Tsiafouli, L. Bjørnlund, J.H. Bracht, M.V. Brady, S. Christensen, P.C. de-Ruiter, T. d’Hertefeldt, J. Frouz, K. Hedlund, L. Hemerikc, H.W.H. Gera, S. Hotes, S.R. Mortimer, H. Setälä, S.P. Sgardelis, K. Uteseny, W.H. van-der-Putten, V. Wolters, and R.D. Bardgett. 2013. Soil food web properties explain ecosystem services across European land use systems. PNAS 110:14296-14301.

Giri, B., P.H. Giang, R. Kumari, R. Prasad, and A. Varma. 2005. Microbial diversity in soils. In: F. Buscot, y A. Varma, editors, Microorganisms in soils: Roles in genesis and functions. Springer-Verlag, Berlin. GER. p. 19-55.

Gliessman, S.R. 2002. Agroecología: procesos ecológicos en agricultura sostenible. CATIE, CRC.

Huising, E.J., R. Coe, J.E. Cares, J.N. Louzada, R. Zanetti, M.S. Moreira, F.X. Susilo, S. Konaté, M. van-Noordwik, and S.P. Huang. 2008. Sampling strategy and design to evaluate below-ground biodiversity. In: M.S. Moreira et al., editors, A handbook of tropical soil biology. Earthscan, London, GBR. p. 17-41.

Kallenbach, C., and A.S. Grandy. 2011. Controls over soil microbial biomass responses to carbon amendments in agricultural systems: A meta-analysis. Agric. Ecosyst. Environ. 144:241-252.

Karyanto, A., C. Rahmadi, E. Franklin, F.X. Susilo, and J. Wellington-de-Morais. 2008. Soil collembola, acari and other mesofauna - The Berlesse method. In: M.S. Moreira et al., editors, A handbook of tropical soil biology. Earthscan, London. GBR. p. 85-94.

Kramer, S., S. Marhan, H. Haslwimmer, L. Ruess, and E. Kandeler. 2013. Temporal variation in surface and subsoil abundance and function of the soil microbial community in an arable soil. Soil Biol. Biochem. 61:76-85. 
Lavelle, P., and A.V. Spain. 2003. Soil ecology. Kluwer Academic Publishers, USA.

Liu, M., F. Hu, X. Chen, Q. Huang, J. Jiao, B. Zhang, and H. Li. 2009. Organic amendments with reduced chemical fertilizer promote soil microbial development and nutrient availability in a subtropical paddy field: The influence of quantity, type and application time of organic amendments. Appl. Soil Ecol. 42:166-175.

Meléndez, G. 2003. Abonos orgánicos: principios, aplicaciones e impacto en la agricultura. UCR, CIA, y CATIE, San José, CRC.

Moore, J.C. 1994. Impact of agricultural practices on soil food web structure: Theory and application. Agric. Ecosyst. Environ. 51:239-247.

Moore, J.C., and H.W. Hunt. 1988. Resource compartmentation and the stability of real food webs. Nature 333:261-263.

Noble, A.D., and S. Ruaysoongnern. 2010. The nature of sustainable agriculture In: G.R. Dixon, and E.L.Tilston, editors, Soil microbiology and sustainable crop production. Springer, USA. p. 1-25.

Orozco, R., y J. Rosales. 2009. Informe de proyecto sobre uso de abonos orgánicos en el cultivo de la mora. Universidad Nacional, CRC.

Orozco, R., y R. Muñoz. 2012. Efecto de abonos orgánicos en las propiedades químicas del suelo y el rendimiento de la mora (Rubus adenotrichus) en dos zonas agroecológicas de Costa Rica. Tecnología en Marcha 25:16-31.

Ortiz, M. 2004. Atlas de Costa Rica [CD]. Instituto Tecnológico de Costa Rica, Cartago, CRC.

Reilly, K., E. Cullen, T. Lola-Luz, D. Stone, J. Valverde, M. Gaffney, N. Brunton, J. Grant, and B.S. Griffiths. 2013. Effect of organic, conventional and mixed cultivation practices on soil microbial community structure and nematode abundance in a cultivated onion crop. J. Sci. Food Agric. 93:3700-3709.

Roveda, G., M. Ramírez, y L. Cabra. 2008. Potencial de microorganismos como biofertilizantes. En: G. Roveda et al., editores, Uso de microorganismos con potencial como biofertilizantes en el cultivo de mora. Corporación Colombiana de Investigación Agropecuaria, COL. p. 44-51.

Scheu, S., L. Ruess, and M. Bonkowski. 2005. Interactions between microorganisms and soil micro- and mesofauna. In: F. Buscot, and A. Varma, editors, Microorganisms in soils: roles in genesis and functions. Springer, GER. p. 253-278.

Singh, B., and Z. Rengel. 2007. The role of crop residues in improving soil fertility. In: P. Marschner, and Z. Rengel, editors, Nutrient cycling in terrestrial ecosystems. Springer-Verlag, Berlin, GER. p.183-206.

Smeding, F.W., and G.R. de-Snoo. 2003. A concept of food-web structure in organic arable farming systems. Landsc. Urban Plan. 65:219-236.

Soil Taxonomy. 1999. A basic system of soil classification for making and interpreting soil surveys. United States Department of Agriculture, USA.

Soto, G. 2003. Abonos orgánicos: definiciones y procesos. En: G. Meléndez et al., editores, Abonos orgánicos: principios, características e impacto en la agricultura. CATIE, UCR y CRC. p. 20-49.

Steel, H., B. Vandecasteele, K. Willekens, K. Sabbe, T. Moens, and W. Bert. 2012. Nematode communities and macronutrients in composts and compost-amended soils as affected by feedstock composition. Appl. Soil Ecol. 61:100-112.

Tilston, E.L., T. Sizmur, G.R. Dixon, W. Otten, and J.A. Harris. 2010. The impact of land-use practices on soil microbes In: G.R. Dixon, and E.L. Tilston, editors, Soil microbiology and sustainable crop production. Springer, USA. p. $273-295$.

van-der-Heijden, G.A., and C. Wagg. 2013. Soil microbial diversity and agro-ecosystem functioning. Plant Soil 363:1-5.

Wardle, D.A. 1999. How soil food webs make plants grow. Trends Ecol. Evol. 14:418-420. 
Wu, Y., Y. Li, C. Zheng, Y. Zhang, and Z. Sun. 2013. Organic amendment application influences soil organism abundance in saline alkali soil. Eur. J. Soil Biol. 54:32-40.

Yeates, G.W., T. Bongers, R.G.M. de-Goede, D.W. Freckman, and S.S. Georgieva. 1993. Feeding habits in soil nematode families and genera - An outline for soil ecologists. J. Nematol. 25:315-331. 